\title{
GLCCI1 is a novel component associated with the PI3K signaling pathway in podocyte foot processes
}

\author{
Sang-Hoon Kim, Hyun-Jung Kim and Chan-Wha Kim
}

Podocyte foot processes are interdigitated to form the slit diaphragm and are crucial for the glomerular filtration barrier. Glucocorticoid-induced transcript 1 (GLCCI1) is transcriptionally regulated, but its signaling pathway in podocytes is unknown. The main objective of this study was to investigate the regulation of podocyte foot process proteins and to investigate the role of GLCCI1 in the phosphoinositide 3-kinase (PI3K) pathway using high glucose-induced podocytes and streptozotocin-induced diabetic rats. In podocytes and rat kidneys, GLCCI1 was found to be highly specific for the glomerulus and podocyte foot processes similar to other podocyte-specific proteins (nephrin, podocin, synatopodin and podocalyxin) based on reverse transcription-PCR, western blotting, immunofluorescence and immunoelectron microscopy analyses. In addition, the decrease in the GLCCI1 expression level under hyperglycemic conditions was restored by treatment with a PI3K inhibitor (wortmannin). Immunofluorescence analysis confirmed that GLCCI1 colocalized with nephrin and synaptopodin both in vivo and in vitro. Finally, immunoelectron microscopy data from streptozotocin-induced diabetic rats showed that GLCCI1 also localized in podocyte foot processes. Hence, GLCCI1 is a component of podocyte foot processes, and its expression appears to be regulated via the PI3K pathway.

Experimental \& Molecular Medicine (2016) 48, e233; doi:10.1038/emm.2016.28; published online 13 May 2016

\section{INTRODUCTION}

Podocytes are major components of the glomerular basement membrane with endothelial cells and play key roles in barrier functions. ${ }^{1}$ Podocytes are terminally differentiated and highly specialized cells composed of cell bodies, major processes and foot processes interlinked by slit diaphragms. ${ }^{2}$ Slit diaphragm proteins are crucial molecular components of the glomerular filtration barrier and are also involved in actin polymerization. ${ }^{3,4}$ Foot process effacement can result from slit diaphragm disruption and podocyte injury. Podocyte injury is a primary factor in the development of diabetic nephropathy. ${ }^{5}$ Therefore, understanding the mechanisms that control the presence of podocyte-specific slit diaphragm proteins and their involvement in diabetic nephropathy may help to provide better treatment.

Many studies have identified novel slit diaphragm proteins in podocyte foot processes. ${ }^{6-9}$ Nephrin is a structural component of the slit diaphragm formed by neighboring podocytes. ${ }^{10,11}$ Podocin is a key factor in the maintenance of slit diaphragm homeostasis. ${ }^{10}$ Synaptopodin is a proline-rich protein that is directly related to actin polymerization. ${ }^{12}$ Podocalyxin plays an essential role in maintaining an open urinary space by virtue of the physicochemical properties of its highly negatively charged ectodomain. ${ }^{13}$ Glucocorticoidinduced transcript 1 (GLCCI1) was first described by Nishibori et al. ${ }^{14}$ and is expressed at the capillary loop stage of glomerular development. The podocyte-specific proteins nephrin and podocin are also expressed in podocytes at the capillary loop stage. ${ }^{15,16}$ GLCCI1, nephrin and podocin have been shown to be simultaneously expressed in podocytes and appear to be activated via the same signaling pathway. In our previous study, we demonstrated that nephrin and podocin expression in the rat glomerulus was regulated by treatment with the phosphoinositide 3-kinase (PI3K) inhibitor wortmannin. ${ }^{17}$ The PI3K family of enzymes is involved in cellular functions such as cell growth, proliferation, differentiation and motility. ${ }^{18} \mathrm{PI} 3 \mathrm{~K}$ is also a critical component of the insulin signaling pathway. All PI3Ks are inhibited by wortmannin, a furanosteroid metabolite derived from the fungi Penicillium funiculosum and Talaromyces (Penicillium) wortmannii and is a specific and covalent inhibitor of PI3Ks. Wortmannin is a common cell biology reagent and has previously been used to inhibit DNA repair, receptormediated endocytosis and cell proliferation. ${ }^{19}$ Treatment of diabetic nephropathy through the PI3K signaling pathway has

Department of Biotechnology, College of Life Sciences and Biotechnology, Korea University, Seoul, Korea

Correspondence: Professor C-W Kim, Department of Biotechnology, College of Life Sciences and Biotechnology, Korea University, 5ga Anam-dong, Sungbuk-ku, Seoul 136-701, Korea.

E-mail: cwkim@korea.ac.kr

Received 17 September 2015; revised 2 December 2015; accepted 7 January 2016 
recently attracted great interest. ${ }^{20,21}$ However, the signaling pathway between GLCCI1 and PI3K in diabetic nephropathy remains unknown. In this study, we demonstrated that GLCCI1 was associated with the PI3K signaling pathway. Serum/glucocorticoid-regulated kinase 1 (SGK1) is activated by PI3K and overexpressed in diabetic nephropathy. Overexpression of SGK1 in diabetic nephropathy inhibits a number of transcription factors, including the cAMP response elementbinding protein (CREB) and forkhead box O3 (FOXO3A). ${ }^{22}$ Therefore, GLCCI1 expression is predicted to be influenced by transcription factors such as FOXO3A.

Here, we demonstrated that GLCCI1 was a novel podocyte foot process protein that was regulated via the PI3K signaling pathway both in vivo and in vitro. Moreover, GLCCI1 appeared to be associated with the development of podocyte foot processes because GLCCI1 colocalized with the known podocyte-specific slit diaphragm proteins nephrin and synaptopodin.

\section{MATERIALS AND METHODS}

\section{Cell culture}

The E11 podocyte cell line (Cell Line Service, Baden-Wuerttemberg, Germany) was used for this study. Differentiated podocytes were cultured in RPMI-1640 medium (Invitrogen, Waltham, MA, USA) containing $10 \%$ fetal bovine serum and penicillin/streptomycin (100 $\mathrm{U} \mathrm{ml}^{-1}$ and $100 \mathrm{mg} \mathrm{ml}^{-1}$, respectively) and incubated at $33^{\circ} \mathrm{C}$ in a $5 \% \mathrm{CO}_{2}$ atmosphere. Podocytes were used between passages 33 and 35. The podocytes were divided into three groups: the normal glucose group (5 mM D-glucose, control), high glucose group ( $25 \mathrm{mM}$ D-glucose, diabetes) and wortmannin-treated diabetic group $(25 \mathrm{~mm}$ D-glucose $+0.32 \mu \mathrm{M}$ wortmannin). Podocytes from the three groups were incubated in culture medium containing either 5 or $25 \mathrm{~mm}$ glucose for $24 \mathrm{~h}$. After $24 \mathrm{~h}$ of incubation, the wortmannin group incubated with high glucose was treated with wortmannin $(0.32 \mu \mathrm{M}$, LC Laboratories, Woburn, MA, USA); the half-maximal inhibitory concentration of wortmannin was determined by CCK-8 assay (data not shown). At $24 \mathrm{~h}$ after treatment with wortmannin, RNA and proteins were extracted from the podocytes.

\footnotetext{
Animals

All animal procedures were performed according to the guidelines provided by the Committee of Animal Ethics at Korea University. Fifteen healthy male 4-week-old Sprague Dawley rats (Orient, Technical Corporation of Charles River Technology, Gapyoung, Korea) weighing $150 \pm 10 \mathrm{~g}$ were randomly divided into 3 groups: the control, diabetic and wortmannin-treated diabetic groups $(n=5$ for each group). Before the experiments, the rats were allowed 1 week to adapt to the laboratory conditions. The rats were maintained under standard conditions with a $12 \mathrm{~h} / 12 \mathrm{~h}$ light/dark cycle at $23 \pm 1{ }^{\circ} \mathrm{C}$ and $50 \pm 10 \%$ humidity. All animals had free access to water. All animals in the diabetic and wortmannin-treated diabetic groups were intraperitoneally injected with $65 \mathrm{mg} \mathrm{kg}^{-1}$ body weight streptozotocin (STZ; Sigma-Aldrich, St Louis, MO, USA) dissolved in $\mathrm{pH} 4.5$ sodium citrate buffer. Control rats were injected with an equivalent volume of sodium citrate buffer. At 3 days after STZ injection, the animals were considered to be diabetic if the plasma glucose levels were $\geqslant 300 \mathrm{mg} \mathrm{dl}^{-1}$. ${ }^{23}$ Wortmannin ( $1 \mathrm{mg} \mathrm{kg}^{-1}$, LC Laboratories) was dissolved in dimethyl sulfoxide and intraperitoneally injected every day for 8 weeks. The control and diabetic group rats were injected
}

with an equivalent volume of dimethyl sulfoxide. All rats were killed after 8 weeks. ${ }^{17}$

\section{Reverse transcription-PCR}

Total RNA was extracted from all specimens (podocytes and Sprague Dawley rats) using the TRIzol reagent (Invitrogen) and reversed transcribed using the SuperScript III reverse transcriptase (Invitrogen). ${ }^{24}$ The sequences of the primers used are as follows: GLCCI1 sense 5'-GCCTTCACCAGTGAAATGACAA-3' and antisense 5'-TTCCTGACCACTTACTGTCCCA-3'; Nephrin sense 5'-GTTCAG CTGGGAGAGACTGG- $3^{\prime}$ and antisense $5^{\prime}$-AATCGGACGACAAG ACGAAC- $3^{\prime}$; Podocin sense 5'-TCTCCCAAACCTGTTGAACC- $3^{\prime}$ and antisense 5'-GCGTGTGTCCATCTTGTGAC-3'; Synaptopodin sense 5'-GCAGAGGAAGTGAGGTCCAG-3' and antisense 5'-GATGCCA CTAGGGTGCTAGG-3'; Podocalxyin sense $5^{\prime}$-GGTGGCAGTGAAG AGATTC- $3^{\prime}$ and antisense $5^{\prime}$-CTGTGAGTCGTTGTTGGTC-3'; and GAPDH sense 5'-CTCTACCCACGGCAAGTTCAA- $3^{\prime}$ and antisense 5'-GGATGACCTTGCCCACAGC-3'. The PCR products were visualized on $1.5 \%$ agarose gels, and the band density was measured using the Gel-Doc image analysis system 2000 (Bio-Rad, Hercules, CA, USA).

\section{Western blotting analysis}

For western blotting, cell lysates of podocytes and rat kidneys were extracted with RIPA buffer (Sigma) containing a protease inhibitor cocktail. Protein concentrations were determined using the Bradford assay method. ${ }^{25}$ For immunoblotting, the extracted protein samples were separated by SDS-polyacrylamide gel electrophoresis and then transferred to nitrocellulose membranes. The membranes were incubated with blocking solution containing a 1:1000 dilution of the rabbit anti-GLCCI1 antibody (ab107491, Abcam, Cambridge, UK), a 1:500 dilution of the goat anti-nephrin antibody (sc-19000, Santa Cruz Biotechnology, Santa Cruz, CA, USA), a 1:500 dilution of the rabbit anti-podocin antibody (sc-21009, Santa Cruz Biotechnology), a 1:1000 dilution of the rabbit anti-SGK1 antibody (ab59337, Abcam) or a 1:1000 dilution of the rabbit anti-FOXO3A antibody (ab47285, Abcam).The membranes were then incubated with blocking solution containing 1:1000 (for GLCCI1, podocin, SGK1 and FOXO3A) dilutions of a horseradish peroxidase-conjugated goat anti-rabbit IgG secondary antibody (1858415, Pierce Biotechnology, Waltham, MA, USA) or a 1:1000 (for nephrin) dilution of a horseradish peroxidaseconjugated donkey anti-goat IgG secondary antibody (ab6885, Abcam). The reactive bands were detected by chemiluminescence using an enhanced chemiluminescence (ECL) system (Millipore, Billerica, MA, USA). Gel bands were scanned with the luminescent image analyzer LAS-3000 and analyzed using the Multi Gauge image analysis software (Fuji Film, Tokyo, Japan). ${ }^{26,27}$

\section{Immunofluorescence}

For the immunofluorescence assay, podocytes and rat kidneys were fixed in $4 \%$ paraformaldehyde. The kidneys were cut into $5 \mu \mathrm{m}$ sections. The cryosections were deparaffinized with xylene and rehydrated using a graded ethanol series. The podocyte cells and kidney sections were blocked with $1 \%$ bovine serum albumin for $1 \mathrm{~h}$, incubated with primary antibodies for $1 \mathrm{~h}$ at room temperature in a humidified chamber, and then incubated with a donkey anti-goat 488-conjugated secondary antibody (ab150129, Abcam) or goat anti-rabbit 568-conjugated secondary antibody (ab175471, Abcam) at room temperature for $1 \mathrm{~h}$. The following antibodies were used: rabbit anti-GLCCI1 (1:100, ab107491, Abcam), goat anti-nephrin (1:100, sc-19000, Santa Cruz Biotechnology), rabbit 
a

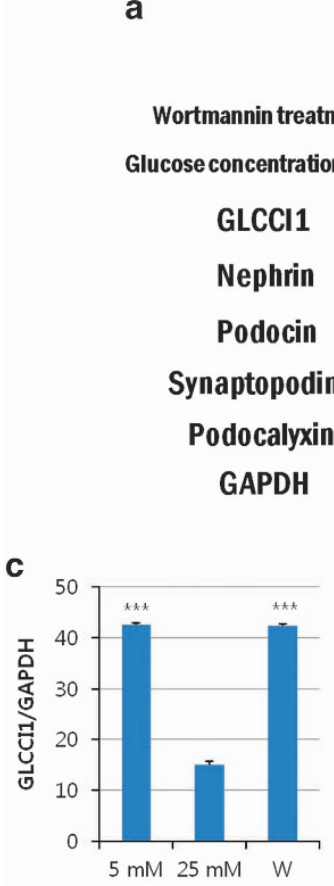

b
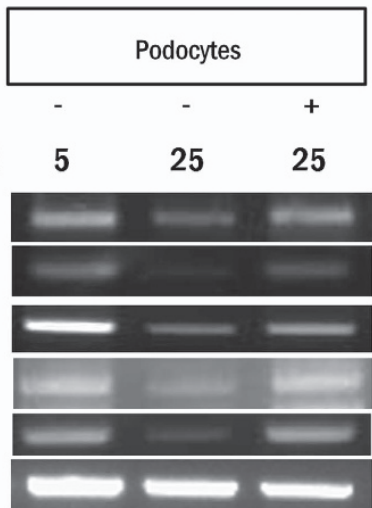

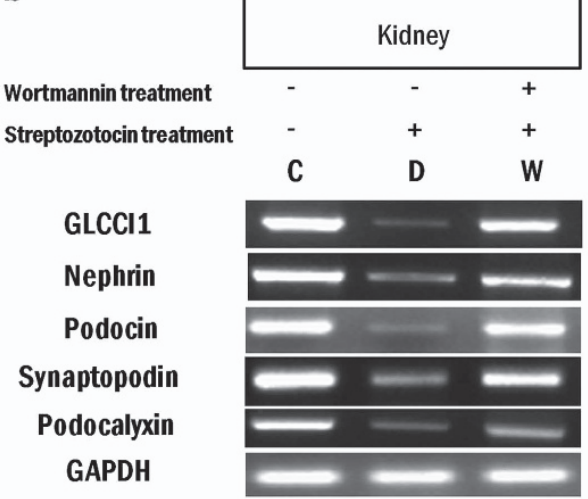

C
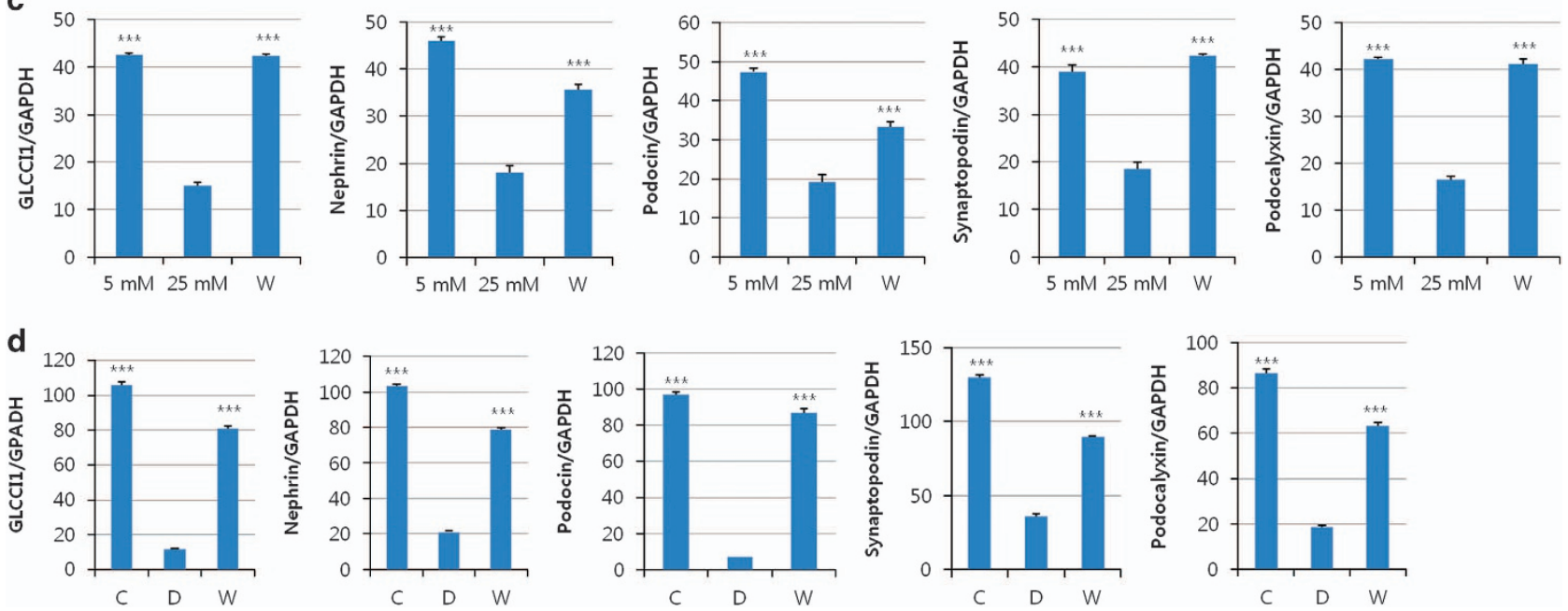

Figure 1 Detection of glucocorticoid-induced transcript 1 (GLCCI1) and podocyte-specific marker gene expression by reverse transcriptionPCR (RT-PCR). (a) Expression of the genes encoding GLCCI1, nephrin, podocin, synaptopodin and podocalyxin showed similar patterns in the podocytes. The expression levels differed significantly between the high glucose-induced and wortmannin-treated diabetic groups of podocytes. (b) The expression of the genes encoding GLCCI1, nephrin, podocin, synaptopodin and podocalyxin showed similar patterns in the kidneys of diabetic rats. In addition, wortmannin ameliorated the expression of all podocyte-specific proteins including GLCCI1. (c, d) The relative band intensity of proteins in podocytes and the kidneys of diabetic rats was observed by RT-PCR. The band intensity was measured using the Multi Gauge V3.0 software (Fuji Film). ${ }^{* * *} P<0.001,5 \mathrm{~mm}$ (control) and wortmannin-treated diabetic groups versus the high glucose-induced group by analysis of variance (ANOVA; means \pm s.e.m., $n=3$ ).

anti-podocin (1:100, sc-21009, Santa Cruz Biotechnology), goat antisynaptopodin (1:100, sc-21536, Santa Cruz Biotechnology), rabbit anti-SGK1 (1:100, ab59337, Abcam) and rabbit anti-FOXO3A (1:100, ab47285, Abcam). All images were obtained using a Carl-Zeiss LSM 700 confocal microscope (Carl-Zeiss, Jena, Germany).

\section{Immunoelectron microscopy}

For immunoelectron microscopy, rat kidney cortex samples were fixed in a solution containing phosphate-buffered $4 \%$ paraformaldehyde (EM grade) and $2.3 \mathrm{~m}$ sucrose overnight. Immunoelectron microscopy was performed according to the method described by Tokuyasu. ${ }^{28}$ In the immunogold labeling experiment, a goat anti-rabbit antibody (gold particles, $10 \mathrm{~nm}$, Abcam) was used to detect GLCCI1. Electron microscopy images were obtained using a Tecnai F20 transmission electron microscope (Don R.Kania, Hillsboro, OR, USA).

\section{Statistical analysis}

All results are expressed as the means \pm s.e.m. Comparison of continuous variables between more than two groups was performed using a one-way ANOVA, and Fisher's least significant difference test was used to assess differences between groups. $P<0.05$ was considered statistically significant. The SPSS software package V12.0 (Chicago, IL, USA) was used for all statistical analyses.

\section{RESULTS}

GLCCI1 is highly specific for the glomerulus and podocyte foot processes and its expression is regulated via the PI3K signaling pathway

The presence of GLCCI1 transcripts in podocytes and rat kidneys was confirmed by reverse transcription-PCR and western blotting. To induce diabetic nephropathy conditions in vitro and in vivo, we used an appropriate high glucose $(25 \mathrm{~mm})$ medium for the culture of podocytes and treated the animals with STZ. At the RNA level, the intensity of the GLCCI1 transcripts was clearly stronger in the normal glucose (5 mM) group of podocytes and the control group of rats. In contrast, a very weak signal was detected in the high glucose- 

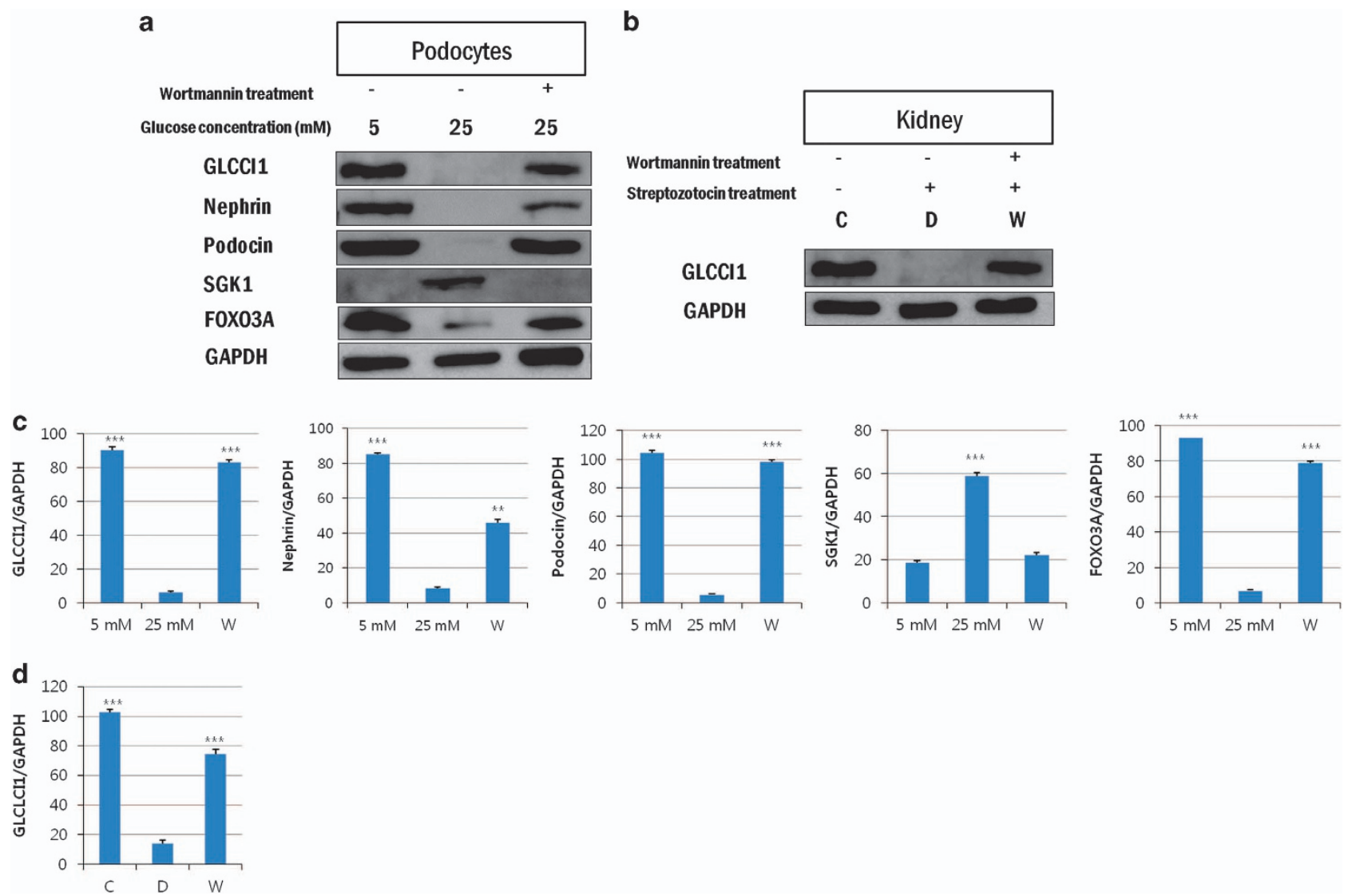

Figure 2 Analysis of glucocorticoid-induced transcript 1 (GLCCI1) protein expression and involvement in the phosphoinositide 3-kinase (PI3K) pathway by western blotting. (a) GLCCI1, nephrin and podocin were expressed only in the 5 mm and the wortmannin-treated diabetic groups. Serum/glucocorticoid-regulated kinase 1 (SGK1) was expressed only in the high glucose-induced group. Forkhead box 03 (FOXO3A) was regulated by wortmannin. (b) GLCCI1 was expressed only in the control and wortmannin-treated diabetic groups. GLCCI1 was significantly decreased in the diabetic group. (c, d) The relative band intensities of proteins in the podocytes and the kidneys of diabetic rats were observed by western blot analysis. The band intensities were measured using the Multi Gauge V3.0 software (Fuji Film). ${ }^{* * *} P<0.001,5 \mathrm{~mm}$ (control) and wortmannin-treated diabetic groups versus the $25 \mathrm{~mm}$ (diabetes) group by analysis of variance (ANOVA; means \pm s.e.m., $n=3$ ).

induced podocytes and STZ-induced diabetic rats. The expression of podocyte-specific markers such as nephrin, podocin, synaptopodin and podocalyxin was similar to GLCCI1 (Figures $1 \mathrm{a}$ and $\mathrm{b}$ ). We also investigated the recovery of GLCCI1 and podocyte-specific proteins at the RNA level following treatment with wortmannin. A CCK-8 assay was used to determine the appropriate wortmannin concentration $(0.32 \mu \mathrm{M})$ for administration to podocytes. In addition, we administered wortmannin to the Sprague Dawley rats at a dosage of $1 \mathrm{mg} \mathrm{kg}^{-1}$. In vitro, a higher-intensity GLCCI1 band was observed in the wortmannin-treated diabetic group, whereas significantly decreased GLCCI1 expression was observed in the high glucose group. Wortmannin promotes GLCCI1 recovery via the PI3K pathway in diabetic nephropathy. Podocyte-specific markers showed expression patterns similar to GLCCI1 in the reverse transcription-PCR (Figure 1a). In addition, we examined whether the GLCCI1 RNA levels were regulated by wortmannin treatment in rat kidneys. GLCCI1 expression in the rat kidneys was regulated by wortmannin treatment. Expression of the podocyte-specific markers nephrin, podocin, synaptopodin and podocalyxin was also recovered in the rat kidneys by wortmannin treatment. GLCCI1 and the podocyte-specific markers showed similar signaling patterns in vivo (Figure $1 \mathrm{~b}$ ).

At the protein level, the GLCCI1 $(58 \mathrm{kDa})$ expression levels differed significantly between the control and diabetic podocytes and rat kidneys. Treatment with wortmannin decreased GLCCI1 expression in the diabetic group (Figures $2 a$ and $b$ ). In the podocytes, nephrin and podocin showed an expression pattern similar to GLCCI1. The expression of the podocytespecific markers nephrin and podocin was confirmed in our previous animal study. ${ }^{17}$ Nephrin and podocin showed a signaling pattern similar to GLCCI1 in vivo. The expression of proteins related to the PI3K pathway was confirmed by western blotting. The SGK1 $(55 \mathrm{kDa})$ and FOXO3A (67 and $105 \mathrm{kDa}$ ) expression levels were significantly different between the 5 and $25 \mathrm{~mm}$ groups of podocytes (Figure 2a). SGK1 is a downstream factor of PI3K that is overexpressed in diabetic nephropathy and regulates a number of transcription factors, such as FOXO3A. ${ }^{22}$ SGK1 was overexpressed in the $25 \mathrm{~mm}$ 
a

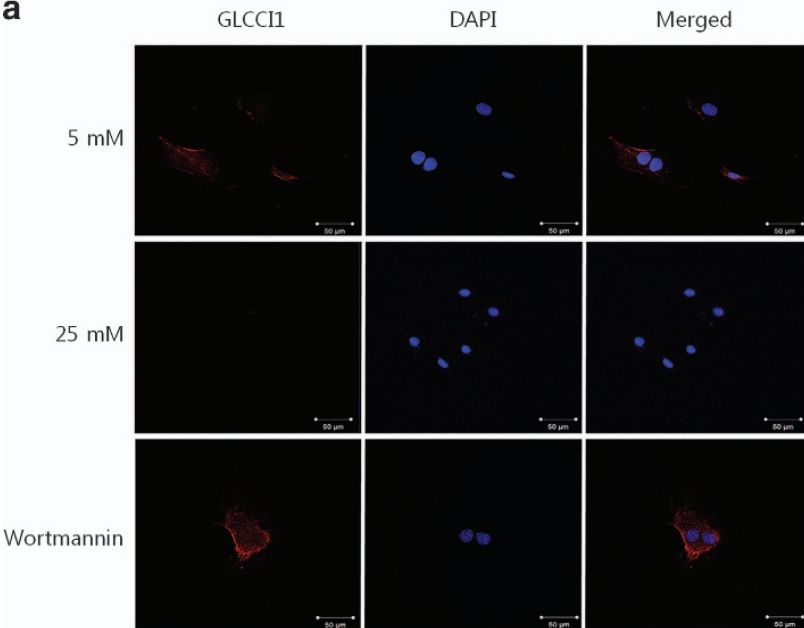

C

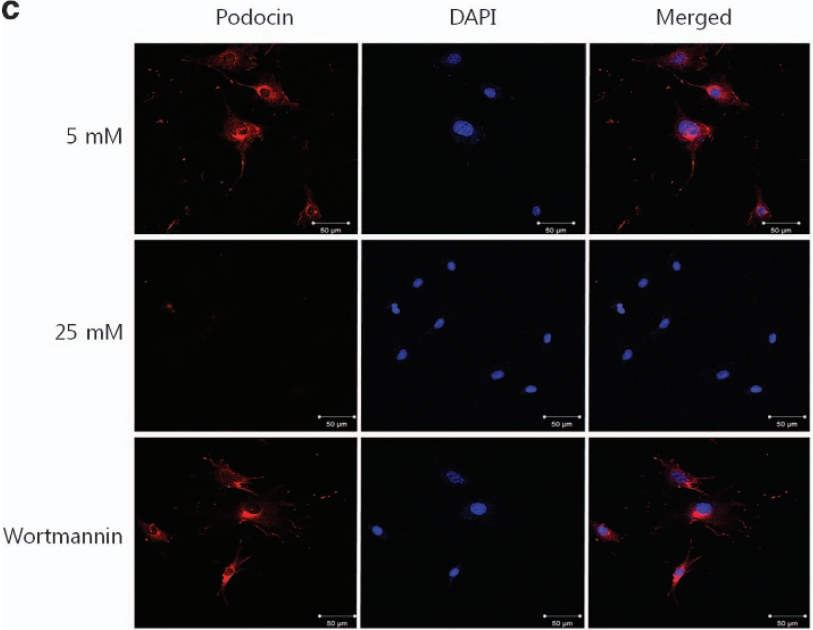

e

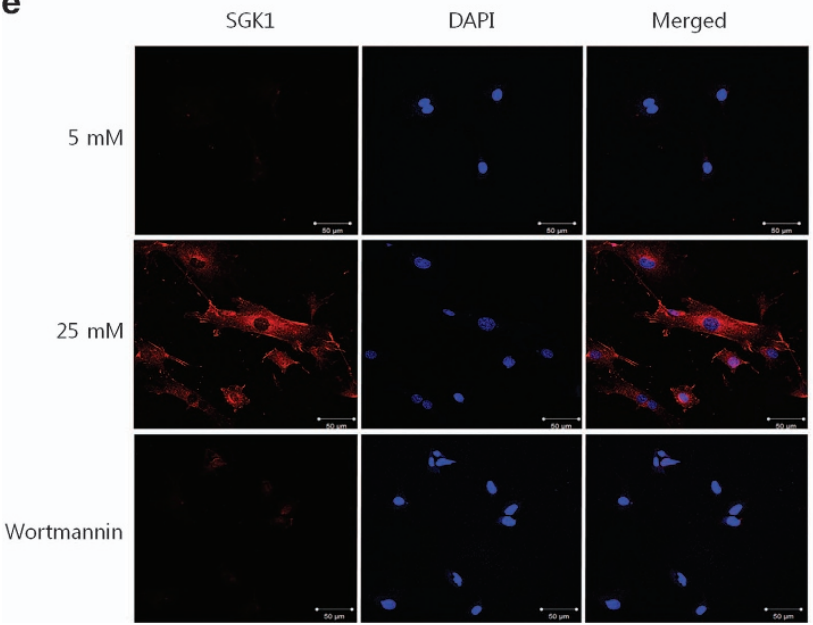

b

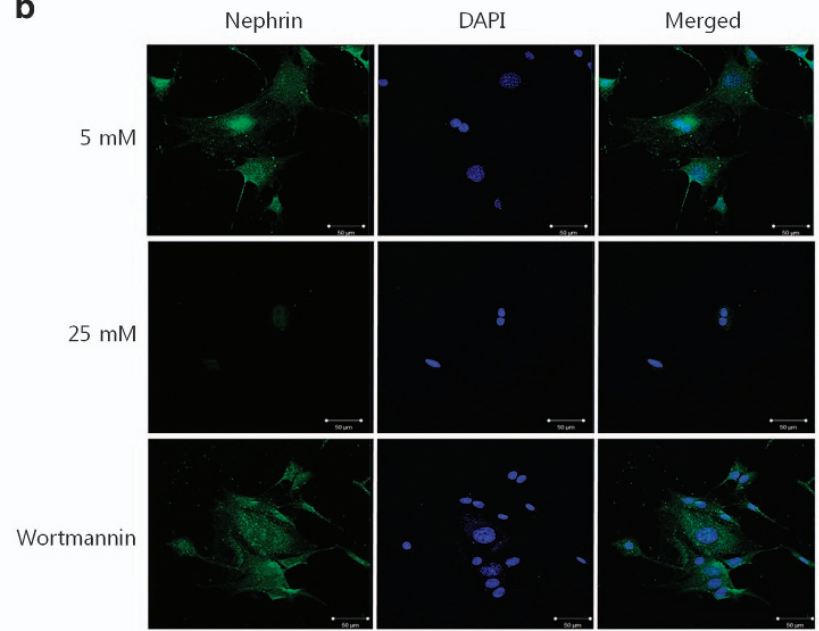

d

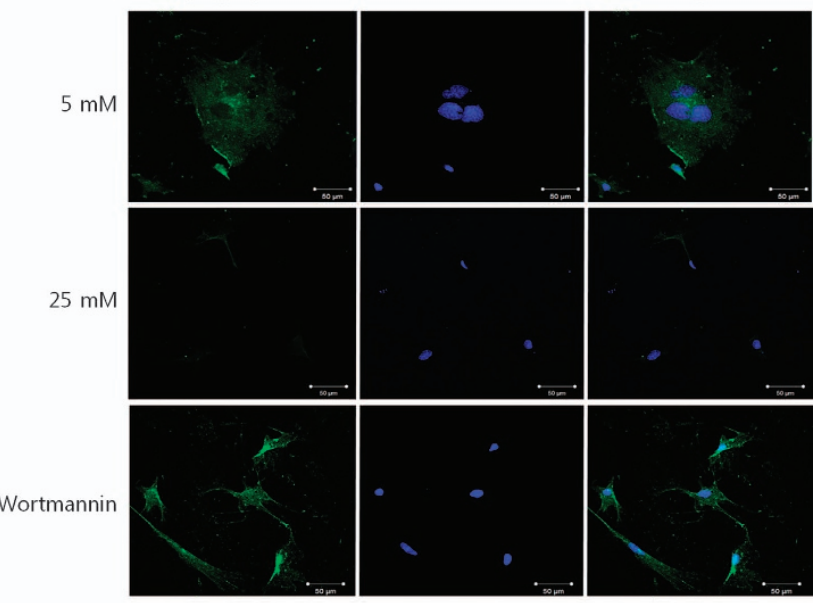

f

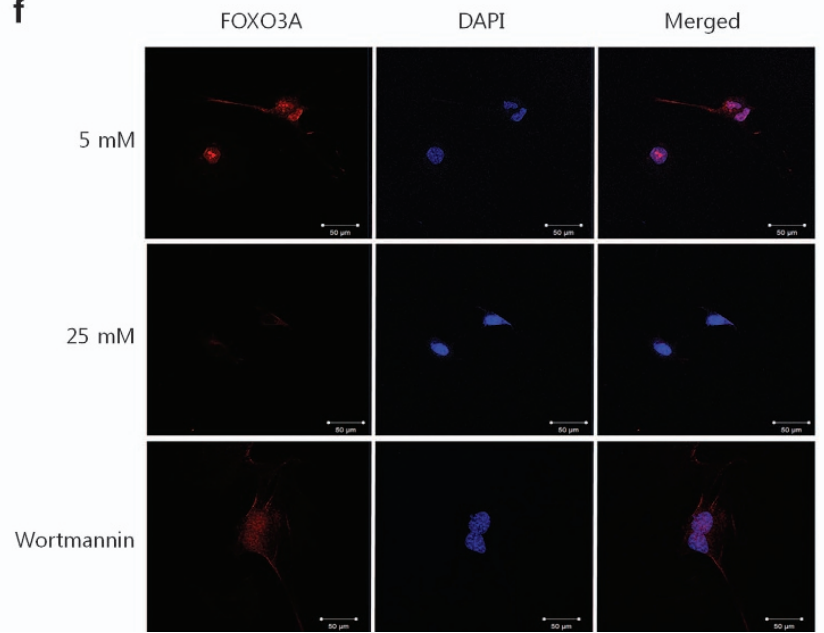

Figure 3 The localization of glucocorticoid-induced transcript 1 (GLCCI1), podocyte-specific markers and proteins involved in the phosphoinositide 3-kinase (PI3K) signaling pathway in podocytes. (a) In podocytes, GLCCI1 (red, Alexa 568 conjugated) was localized in the 4',6-diamidino-2-phenylindole (DAPI)-stained nuclei. No GLCCI1 localization was observed in the 25 mm D-glucose-treated podocytes. However, GLCCI1 was regulated by wortmannin treatment. (b-d) The podocyte-specific proteins nephrin (green, Alexa 488 conjugated), podocin (red) and synaptopodin (green) showed a reactivity pattern similar to GLCCI1 in podocytes treated with wortmannin. (e) Serum/ glucocorticoid-regulated kinase 1 (SGK1; red) was observed only in the $25 \mathrm{~mm}$ group. We confirmed that treatment with wortmannin decreased SGK1 expression. (f) Forkhead box 03 (FOXO3A; red) was observed in the 5 mm group. A lower FOXO3A signal was accompanied by high reactivity of SGK1 in the $25 \mathrm{~mm}$ group. Localization of FOXO3A was regulated by treatment with wortmannin. Original magnification: $\times 400$. Scale bar, $50 \mu \mathrm{m}$. 
a

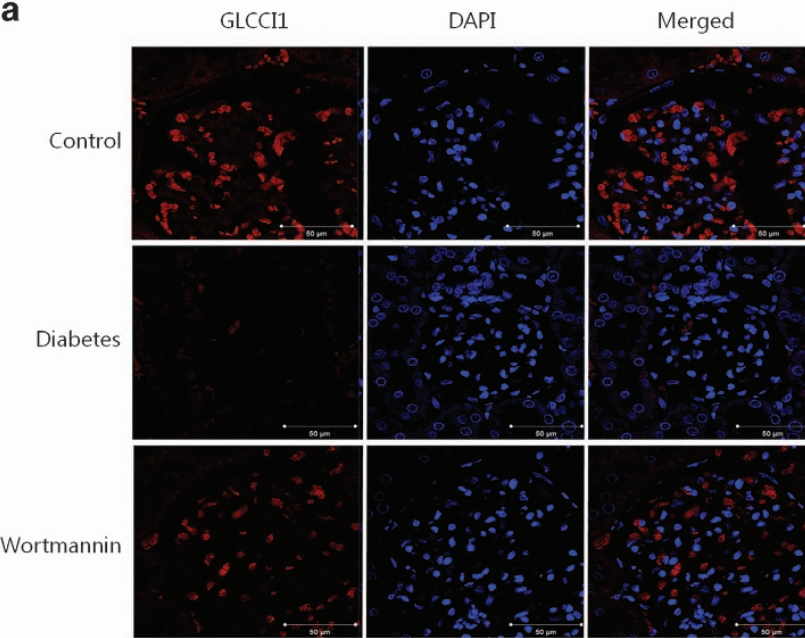

C

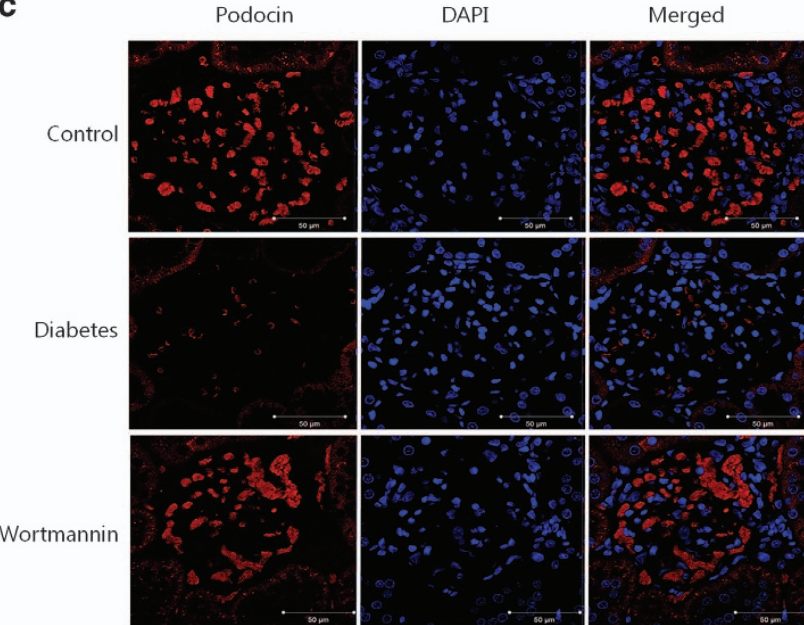

e

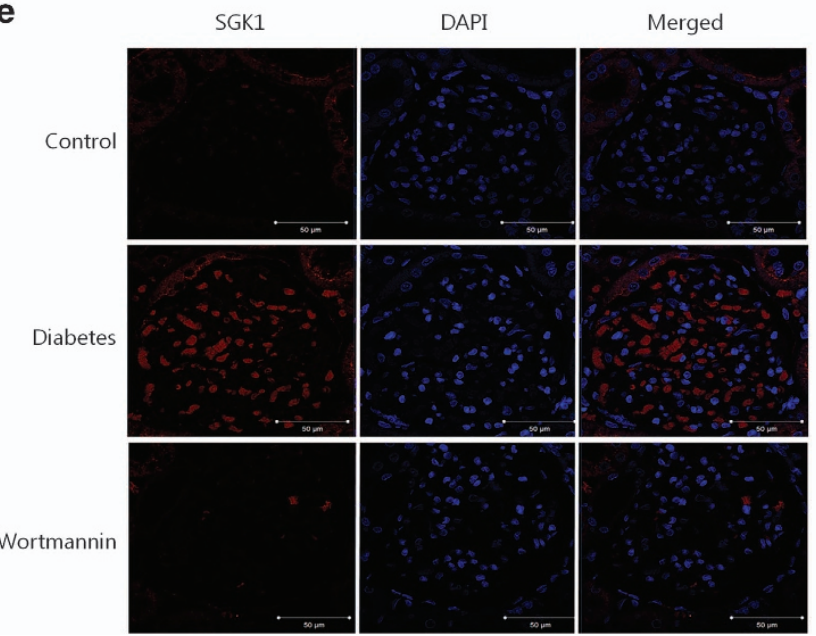

b

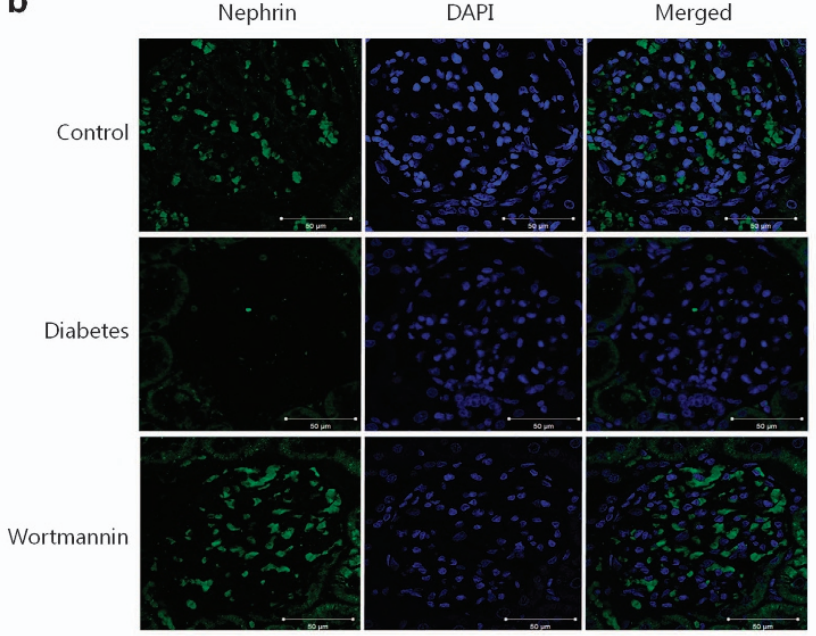

d

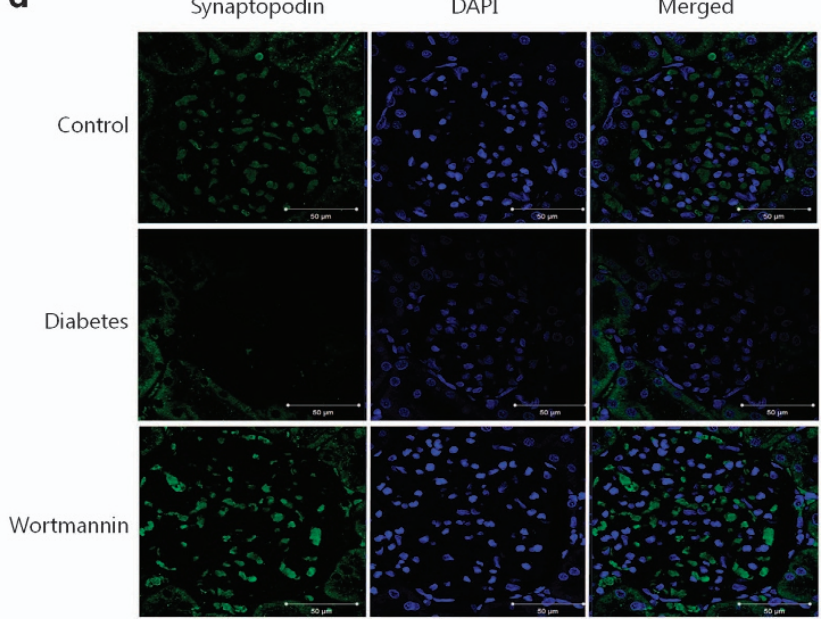

f

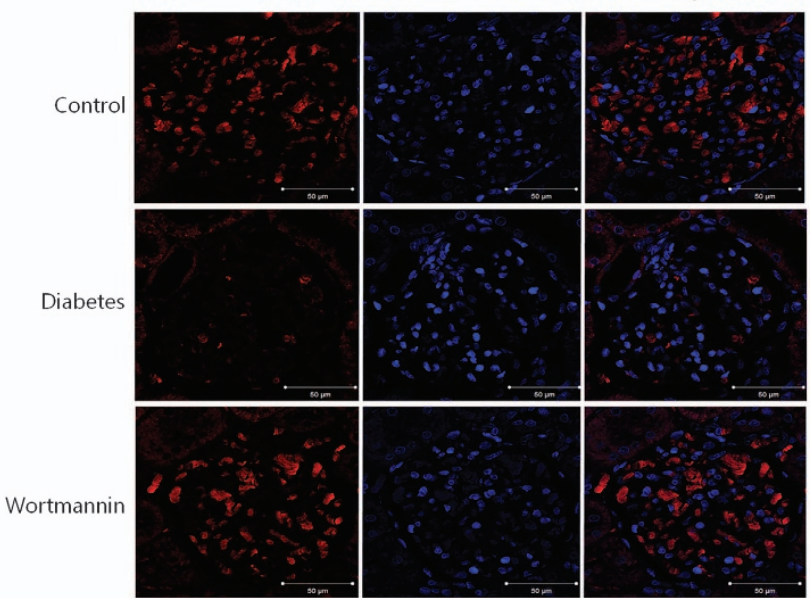

Figure 4 The localization of glucocorticoid-induced transcript 1 (GLCCI1), podocyte-specific markers and proteins associated with the phosphoinositide 3-kinase (PI3K) signaling pathway in the rat glomerulus. (a) Staining of rat kidney sections with an antibody against GLCCI1 (red) is shown. The diabetic group showed low reactivity. However, GLCCI1 was regulated by wortmannin treatment. (b-d) The podocyte-specific proteins nephrin (green), podocin (red) and synaptopodin (green) showed a reactivity pattern similar to GLCCI1 following wortmannin treatment. (e) Serum/glucocorticoid-regulated kinase 1 (SGK1; red) was observed only in the diabetic group. We confirmed that SGK1 expression was decreased by wortmannin. (f) Forkhead box 03 (FOXO3A; red) was observed in the control group. The lower reactivity of FOXO3A was accompanied by the overexpression of SGK1 in the diabetic group. The localization of FOXO3A was regulated by wortmannin treatment. Original magnification: $\times 400$. Scale bar, $50 \mu \mathrm{m}$. 
a

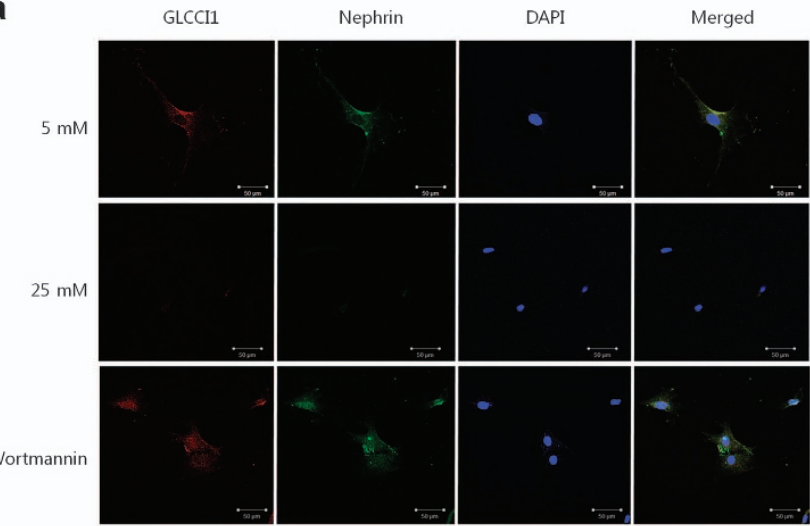

C

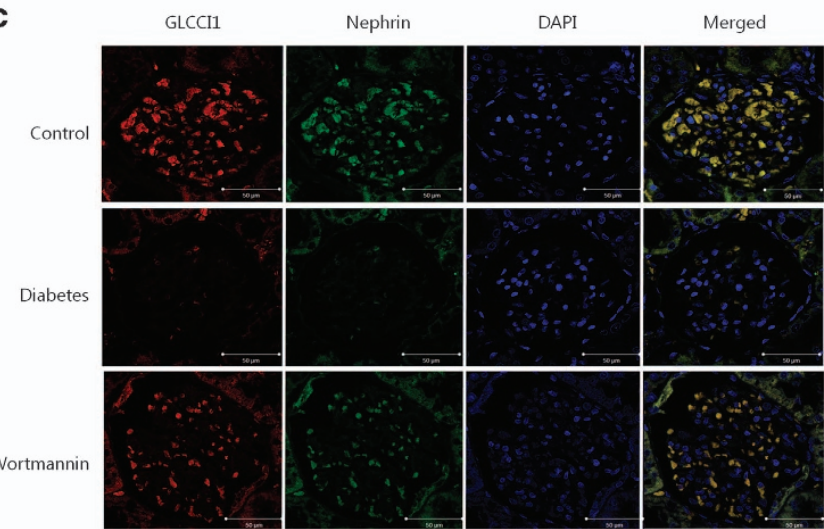

b

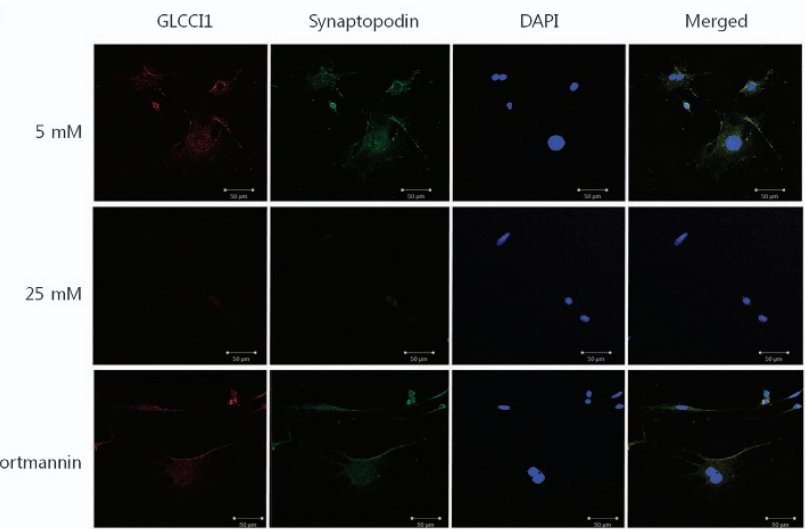

d

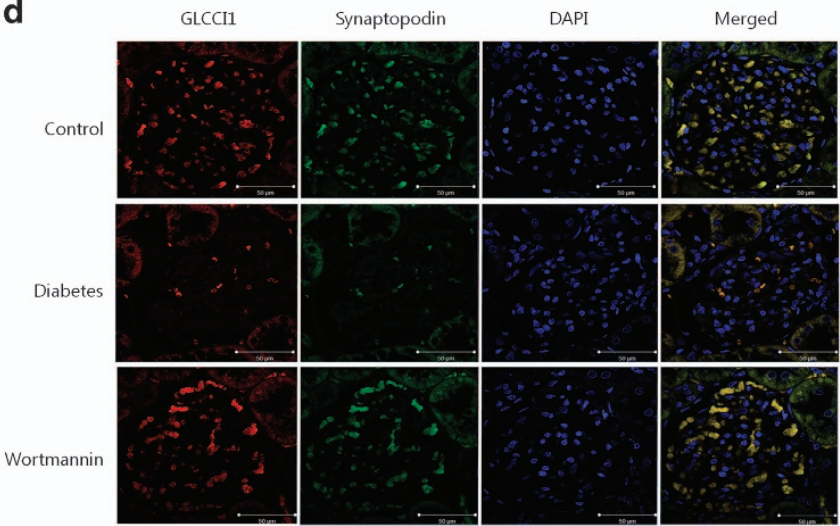

Figure 5 Colocalization of glucocorticoid-induced transcript 1 (GLCCI1) with the podocyte-specific proteins nephrin and synaptopodin. (a) Double labeling with the podocyte-specific marker nephrin (green) showed a complete overlap (merge, yellow) with the distribution of GLCCI1 (red) in the $5 \mathrm{~mm}$ and wortmannin-treated diabetic groups of cultured podocytes. (b) GLCCI1 (red) also colocalized with synaptopodin (green). We observed overlapping reactivity (yellow indicates red plus green) in the 5 mm and wortmannin-treated diabetic groups of cultured podocytes. (c, d) In rat glomeruli, we observed overlapping reactivity (yellow) via the colocalization of GLCCI1 (red) with nephrin and synaptopodin (green). Wortmannin ameliorated the lower reactivity in glomeruli from rats in the diabetic group. Original magnification: $\times 400$. Scale bar, $50 \mu \mathrm{m}$.

group and was downregulated by treatment with wortmannin. FOXO3A expression, which was blocked by the overexpression of SGK1 in the $25 \mathrm{~mm}$ group, was also regulated by wortmannin. Finally, GLCCI1 expression was regulated by the increased FOXO3A expression in the wortmannin-treated diabetic group. All of the results demonstrated statistical significance by the SPSS software (Figures $1 c, d$ and $2 c, d$ ).

Taken together, these results demonstrated that GLCCI1 was expressed with nephrin, podocin, synaptopodin and podocalyxin in vivo and in vitro and that GLCCI1 was regulated by a signaling pathway associated with PI3K in diabetic nephropathy.

\section{Localization of GLCCI1 in cultured podocytes and rat glomeruli}

We investigated the localization of GLCCI1 in cultured podocytes and rat glomeruli via immunofluorescence. Immunofluorescent staining with an anti-GLCCI1 antibody (Alexa Fluor 568, red, Alan Hirzel, Cambridge, MA, USA) revealed intense reactivity in cultured podocytes and rat glomeruli. In the $25 \mathrm{~mm}$ group of podocytes and the diabetic group of animals, very weak reactivity was observed in contrast to the wortmannin-treated diabetic group (Figures $3 a$ and $4 a$ ). GLCCI1 was localized in the cultured podocytes and rat glomeruli. Therefore, the localization of podocyte-specific markers was examined to determine similarities with GLCCI1. Nephrin (Alexa Fluor 488, green), podocin (Alexa Fluor 568, red) and synaptopodin (Alexa Fluor 488, green) showed patterns of reactivity similar to GLCCI1 (Figures $3 \mathrm{~b}-\mathrm{d}$ and 4b-d). As shown in Figures 3 and 4, wortmannin ameliorated the weak reactivity observed in the disease conditions. Using immunofluorescence, we also confirmed that SGK1 (Alexa Fluor 568, red) and FOXO3A (Alexa Fluor 568, red) were associated with the PI3K pathway. SGK1 was observed only in the $25 \mathrm{~mm}$ group of podocytes and the diabetic group of animals (Figures 3e and 4e). Because SGK1 was overexpressed in diabetic nephropathy, it was not observed in the control and wortmannin-treated diabetic groups in vitro and in vivo. In contrast, FOXO3A was observed only in the control and wortmannin-treated diabetic groups. No FOXO3A reactivity was observed in the $25 \mathrm{~mm}$ group of podocytes and the diabetic group of animals (Figures $3 \mathrm{f}$ and $4 \mathrm{f}$ ). Because FOXO3A is a 

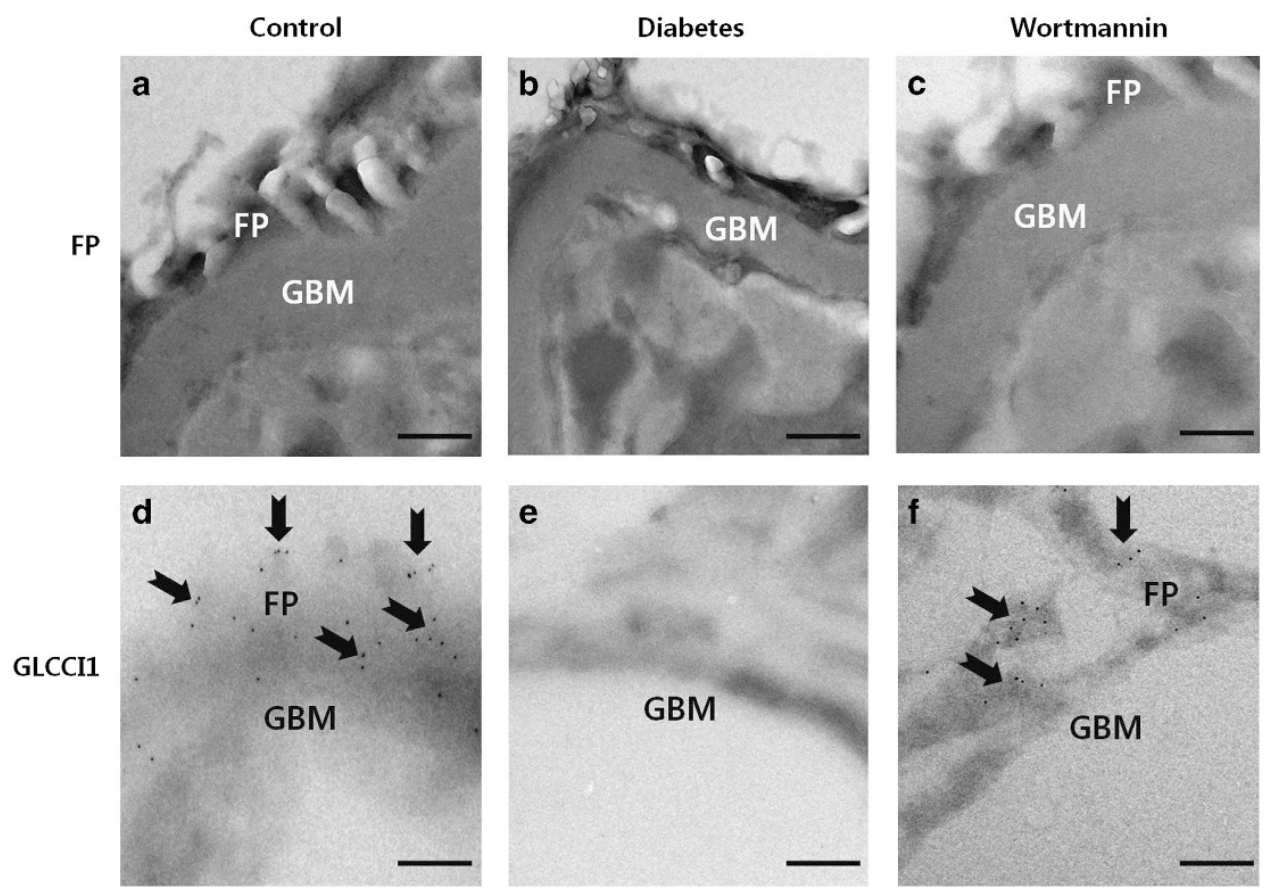

Figure 6 Localization of glucocorticoid-induced transcript 1 (GLCCI1) in podocyte foot processes (FPs) by immunoelectron microscopy. (a) FPs formed by normal podocytes in the control group. (b) Effacement of FPs by podocyte injury in the diabetic group. Podocytes were disrupted by streptozotocin (STZ) treatment. (c) Podocyte FPs were regulated around the glomerular basement membrane (GBM) by treatment with wortmannin. Wortmannin could restore podocytes to their original condition. (d) Immunogold particles (arrowheads) marking GLCCI1 (10 nm gold) were observed in podocyte FPs of the control group. (e) No labeling was observed in podocyte FPs of the diabetic group. (f) Localization of GLCCI1 in podocyte FPs was observed in the wortmannin-treated diabetic group. Scale bar, $200 \mathrm{~nm}$.

Table 1 Summary of quantitative immunoelectron microscopy data

\begin{tabular}{lcc}
\hline Group & FP number per $\mu m$ & Gold particle per $\mu m^{2}$ \\
\hline Control & $8.5 \pm 0.6$ & $30.3 \pm 4.3$ \\
Diabetes & $0.3 \pm 0.2^{* * *}$ & $2.5 \pm 0.8^{* * *}$ \\
Wortmannin & $6.9 \pm 0.5$ & $19.7 \pm 3.9$ \\
\hline
\end{tabular}

Abbreviation: FP, foot process.

The quantitation of foot process formation (foot process number per $\mu \mathrm{m}$ ) and the gold labeling intensity of glucocorticoid-induced transcript 1 (GLCCI1; gold particle per $\mu \mathrm{m}^{2}$ ) are shown.

$* * * P<0.001$, control and wortmannin-treated diabetic groups versus the diabetes group by analysis of variance (ANOVA; means \pm s.e.m., $n=3$ ).

transcription factor, its activation is associated with translocation to the nucleus. Thus, FOXO3A must affect the expression and localization of GLCCI1 through the PI3K pathway.

These findings suggest that GLCCI1 is localized with podocyte-specific markers in cultured podocytes and rat glomeruli and that GLCCI1 is regulated by SGK1 and FOXO3A via the PI3K pathway in diabetic nephropathy.

\section{GLCCI1 colocalizes with nephrin and synaptopodin}

We performed double-labeling immunofluorescence to examine the overlap between GLCCI1 and the podocytespecific markers nephrin and synaptopodin in cultured podocytes and rat glomeruli. GLCCI1 colocalized with nephrin and synaptopodin in the cultured podocytes. Merged images showed that the GLCCI1 staining pattern completely overlapped with nephrin and synaptopodin in the cultured podocytes (Figures 5a and b). GLCCI1 also colocalized with nephrin and synaptopodin in the kidney glomeruli; indeed, the localization of GLCCI1 showed a complete overlap with nephrin and synaptopodin in the rat glomeruli (Figures $5 \mathrm{c}$ and d). In the $25 \mathrm{~mm}$ and diabetic groups, no reactivity of GLCCI1 with nephrin and synaptopodin was observed, whereas increased reactivity of GLCCI1 with nephrin and synaptopodin was observed following treatment with wortmannin.

These data show that GLCCI1, nephrin and synaptopodin are simultaneously regulated via the PI3K pathway. Nephrin is present in foot processes; ${ }^{15}$ therefore, GLCCI1 must also be present in foot processes. It is important to note that GLCCI1 is involved in process development.

\section{GLCCI1 expression in the foot processes of rat glomeruli}

To clarify whether GLCCI1 was expressed and localized in the foot processes of podocytes, rat glomeruli were examined using electron microscopy. Electron microscopic examination of kidneys from the control group rats revealed the complete foot process morphology (Figure 6a). In the STZ-induced diabetic rats, effacement of the foot processes was observed because of podocyte injury by STZ (Figure 6b). The morphology of the disrupted foot processes in the diabetic group was recovered by wortmannin treatment (Figure 6c). In the immunoelectron microscopy images, the GLCCI1 label was detected in the podocyte foot processes in the control group (Figure 6d). In contrast, GLCCI1 was absent from the podocyte 
foot processes in the glomeruli from the diabetic group (Figure 6e). Administration of wortmannin increased GLCCI1 expression in the podocyte foot processes of the glomerulus via the PI3K pathway (Figure 6f). The quantitation of foot processes and gold-labeled GLCCI1 showed that the expression was significantly decreased in rats with STZ-induced diabetes, whereas the foot processes and gold-labeled GLCCI1 was restored in the wortmannin-treated rats compared with the controls (Table 1). The electron microscopy analyses demonstrated that GLCCI1 was expressed in podocyte foot processes via the PI3K pathway.

\section{DISCUSSION}

GLCCI1 expression has been detected in diverse tissues, including kidney glomeruli. ${ }^{14}$ However, the signaling pathway associated with GLCCI1 expression in kidney podocytes remains unknown. In this study, we demonstrated for the first time that GLCCI1 was specifically expressed in podocyte foot processes via the PI3K pathway in vivo and in vitro. To explore the regulation of GLCCI1 under hyperglycemic conditions, we used two models for in vitro and in vivo studies: a high glucoseinduced podocyte cell line and STZ-induced rats, respectively. In all specimens, GLCCI1 was expressed exclusively in the control and wortmannin-treated diabetic groups and was regulated by the PI3K pathway. A similar expression pattern was observed for podocyte-specific proteins such as nephrin, podocin, synaptopodin and podocalyxin that are important for the glomerular filtration barrier. We investigated changes in the protein expression of GLCCI1, SGK1 and FOXO3A, which are associated with the PI3K pathway, in the cultured podocytes. SGK1 is activated by PI3K and overexpressed in diabetic nephropathy. ${ }^{22}$ In addition, SGK1 regulates a number of transcription factors such as CREB and FOXO3A. FOXO3A was highly expressed in the wortmannin-treated diabetic group of podocytes and was accompanied by the downregulation of SGK1 in the wortmannin-treated diabetic group. This signaling pathway can induce GLCCI1 expression because GLCCI1 is a transcript protein. However, our data showed that the podocyte-specific markers nephrin and podocin (but not a transcript protein) showed expression patterns similar to GLCCI1 and were also regulated via the PI3K pathway. This finding indicates that GLCCI1 and the podocyte-specific proteins nephrin, podocin, synaptopodin and podocalyxin are coregulated via the PI3K pathway.

In this study, we confirmed that GLCC1 was localized to podocytes and rat glomeruli similar to other podocyte-specific proteins (nephrin, podocin, synatopodin and podocalyxin). In contrast, extremely low reactivity of GLCCI1 and podocytespecific proteins was detected under hyperglycemic conditions. In addition, both podocyte-specific proteins and GLCC1 were found to be regulated by treatment with wortmannin. Colocalization of SGK1 and FOXO3A, which are associated with the PI3K signaling pathway, was also observed in vivo and in vitro. This result supports the hypothesis that GLCCI1 is localized in the podocyte and rat glomerulus and that its localization is related to PI3K signaling.
In the colocalization studies, the GLCCI1 expression pattern showed a complete overlap with the podocyte-specific proteins nephrin and synaptopodin in the $5 \mathrm{~mm}$ group of cultured podocytes and the control group of rat glomeruli. No reactivity was observed in the $25 \mathrm{~mm}$ group of cultured podocytes and the diabetic group of rat glomeruli. Wortmannin increased the reactivity of GLCCI1 and the podocyte-specific proteins nephrin and synaptopodin in vivo and in vitro. GLCCI1 was found to be regulated simultaneously with nephrin and synaptopodin. Nephrin is a key factor in the glomerular slit diaphragm..$^{29}$ The absence of nephrin leads to proteinuria and foot process effacement. ${ }^{30}$ GLCCI1 deficiency also leads to proteinuria. ${ }^{14}$ Synaptopodin is essential for the integrity of the podocyte actin cytoskeleton and the regulation of podocyte cell migration. ${ }^{31}$ Synaptopodin protects against proteinuria in kidney podocytes. ${ }^{32}$ Thus, GLCCI1 in addition to nephrin and synaptopodin is a major factor in podocyte foot process functions.

The immunoelectron microscopy results showed a significant decrease in GLCCI1 expression and foot process effacement in rats in the diabetic group. Moreover, we directly confirmed the expression and localization of GLCCI1 in podocyte foot processes and the glomerular basement membrane by treatment with wortmannin. Foot process effacement was not detected following wortmannin treatment in the diabetic group. Wortmannin may regulate foot process effacement and decrease GLCCI1 in the diabetic group. This result indicates that GLCCI1 is related to the PI3K signaling pathway in podocytes.

In conclusion, we identified GLCCI1 as a novel podocyte foot process protein that was regulated via the PI3K pathway. GLCCI1 colocalizes with nephrin and synaptopodin that are known podocyte slit diaphragm proteins. GLCCI1 may play an important role in the proper formation of foot processes in podocytes. The presence of GLCCI1 in association with the PI3K signaling pathway may contribute to development and maintenance of podocyte structure and function.

\section{CONFLICT OF INTEREST}

The authors declare no conflict of interest.

\section{ACKNOWLEDGEMENTS}

This study was supported by a Korea University Grant and a grant from BK21 plus (Brain Korea 21 plus). We thank the Center for Analytical and Lifescience Instruments at Korea University for providing the confocal microscope. We also thank Dr Sung-Soo Kim of the Advanced Analysis Center at the Korea Institute of Science and Technology (KIST) for providing the electron microscope.

\footnotetext{
1 Li JJ, Kwak SJ, Jung DS, Kim JJ, Yoo TH, Ryu DR et al. Podocyte biology in diabetic nephropathy. Kidney Int Suppl 2007; 72: S36-S42.

2 Mundel P, Kriz W. Structure and function of podocytes: an update. Anat Embryol (Berl) 1995; 192: 385-397.

3 New LA, Martin CE, Jones N. Advances in slit diaphragm signaling. Curr Opin Nephrol Hypertens 2014; 23: 420-430.
} 
4 Pavenstadt $\mathrm{H}$, Kriz W, Kretzler M. Cell biology of the glomerular podocyte. Physiol Rev 2003; 83: 253-307.

5 Reddy GR, Kotlyarevska K, Ransom RF, Menon RK. The podocyte and diabetes mellitus: is the podocyte the key to the origins of diabetic nephropathy? Curr Opin Nephrol Hypertens 2008; 17: 32-36.

6 Endlich N, Schordan E, Cohen CD, Kretzler M, Lewko B, Welsch T et al. Palladin is a dynamic actin-associated protein in podocytes. Kidney Int 2009; 75: 214-226.

7 Harita Y, Kurihara H, Kosako H, Tezuka T, Sekine T, Igarashi $T$ et al. Neph1, a component of the kidney slit diaphragm, is tyrosinephosphorylated by the Src family tyrosine kinase and modulates intracellular signaling by binding to Grb2. J Biol Chem 2008; 283: 9177-9186.

8 Hirabayashi S, Mori H, Kansaku A, Kurihara H, Sakai T, Shimizu F et al. MAGI-1 is a component of the glomerular slit diaphragm that is tightly associated with nephrin. Lab Invest 2005; 85: 1528-1543.

9 Sistani L, Duner F, Udumala S, Hultenby K, Uhlen M, Betsholtz C et al. Pdlim2 is a novel actin-regulating protein of podocyte foot processes. Kidney Int 2011; 80: 1045-1054.

10 Perysinaki GS, Moysiadis DK, Bertsias G, Giannopoulou I, Kyriacou K, Nakopoulou $L$ et al. Podocyte main slit diaphragm proteins, nephrin and podocin, are affected at early stages of lupus nephritis and correlate with disease histology. Lupus 2011; 20: 781-791.

11 Ruotsalainen V, Ljungberg P, Wartiovaara J, Lenkkeri U, Kestila M, Jalanko $\mathrm{H}$ et al. Nephrin is specifically located at the slit diaphragm of glomerular podocytes. Proc Natl Acad Sci USA 1999; 96: 7962-7967.

12 Srivastava T, Garola RE, Whiting JM, Alon US. Synaptopodin expression in idiopathic nephrotic syndrome of childhood. Kidney Int 2001; 59: $118-125$.

13 Takeda T. Podocyte cytoskeleton is connected to the integral membrane protein podocalyxin through $\mathrm{Na}+/ \mathrm{H}+$-exchanger regulatory factor 2 and ezrin. Clin Exp Nephrol 2003; 7: 260-269.

14 Nishibori Y, Katayama K, Parikka M, Oddsson A, Nukui M, Hultenby K et al. Glccil deficiency leads to proteinuria. J Am Soc Nephrol 2011; 22: 2037-2046.

15 Holzman LB St, John PL, Kovari IA, Verma R, Holthofer H, Abrahamson DR. Nephrin localizes to the slit pore of the glomerular epithelial cell. Kidney Int 1999; 56: 1481-1491.

16 Roselli S, Gribouval O, Boute N, Sich M, Benessy F, Attie T et al. Podocin localizes in the kidney to the slit diaphragm area. Am J Pathol 2002; 160: 131-139.

17 Kim SH, Jang YW, Hwang P, Kim HJ, Han GY, Kim CW. The reno-protective effect of a phosphoinositide 3-kinase inhibitor wortmannin on streptozotocin-induced proteinuric renal disease rats. Exp Mol Med 2012; 44: 45-51.

18 Yousif $\mathrm{MH}$. Phosphoinositide 3-kinase contributes to diabetes-induced abnormal vascular reactivity in rat perfused mesenteric bed. Cell Biochem Funct 2008; 26: 451-458.

19 Liu Y, Shreder KR, Gai W, Corral S, Ferris DK, Rosenblum JS. Wortmannin, a widely used phosphoinositide 3-kinase inhibitor, also potently inhibits mammalian polo-like kinase. Chem Biol 2005; 12: 99-107.

20 Ha TS, Hong EJ, Han GD. Diabetic conditions downregulate the expression of CD2AP in podocytes via PI3-K/Akt signalling. Diabetes Metab Res Rev 2015; 31: 50-60.
21 Zhu J, Sun N, Aoudjit L, Li H, Kawachi H, Lemay S et al. Nephrin mediates actin reorganization via phosphoinositide 3-kinase in podocytes. Kidney Int 2008; 73: 556-566.

22 Lang F, Gorlach A, Vallon V. Targeting SGK1 in diabetes. Expert Opin Ther Targets 2009; 13: 1303-1311.

23 Lee JJ, Yi HY, Yang JW, Shin JS, Kwon JH, Kim CW. Characterization of streptozotocin-induced diabetic rats and pharmacodynamics of insulin formulations. Biosci Biotechnol Biochem 2003; 67: 2396-2401.

$24 \mathrm{Hwang}$ PT, Kwon OD, Kim HJ, Kim BG, Kim SH, Jang YW et al. Hyperglycemia decreases the expression of ATP synthase beta subunit and enolase 2 in glomerular epithelial cells. Tohoku J Exp Med 2013; 231: $45-56$.

25 Field A, Field J. Melamine and cyanuric acid do not interfere with Bradford and Ninhydrin assays for protein determination. Food Chem 2010; 121: 912-917.

26 Cho EH, Kim MR, Kim HJ, Lee DY, Kim PK, Choi KM et al. The discovery of biomarkers for type 2 diabetic nephropathy by serum proteome analysis. Proteomics Clin Appl 2007; 1: 352-361.

$27 \mathrm{Kim} \mathrm{HJ}$, Cho EH, Yoo JH, Kim PK, Shin JS, Kim MR et al. Proteome analysis of serum from type 2 diabetics with nephropathy. J Proteome Res 2007; 6: 735-743.

28 Loussert Fonta C, Leis A, Mathisen C, Bouvier DS, Blanchard W, Volterra A et al. Analysis of acute brain slices by electron microscopy: a correlative light-electron microscopy workflow based on Tokuyasu cryo-sectioning. J Struct Biol 2015; 189: 53-61.

29 Done SC, Takemoto M, He L, Sun Y, Hultenby K, Betsholtz C et al. Nephrin is involved in podocyte maturation but not survival during glomerular development. Kidney Int 2008; 73: 697-704.

30 Menne J, Meier M, Park JK, Boehne M, Kirsch T, Lindschau C et al. Nephrin loss in experimental diabetic nephropathy is prevented by deletion of protein kinase C alpha signaling in-vivo. Kidney Int 2006; 70: $1456-1462$.

31 Asanuma K, Yanagida-Asanuma E, Faul C, Tomino Y, Kim K, Mundel P. Synaptopodin orchestrates actin organization and cell motility via regulation of RhoA signalling. Nat Cell Biol 2006; 8: 485-491.

32 Yanagida-Asanuma E, Asanuma K, Kim K, Donnelly M, Young Choi H, Hyung Chang $J$ et al. Synaptopodin protects against proteinuria by disrupting Cdc42:IRSp53:Mena signaling complexes in kidney podocytes. Am J Pathol 2007; 171: 415-427.

(c) (1) $\circledast \Theta$ This work is licensed under a Creative Commons Attribution-NonCommercial-NoDerivs 4.0 International License. The images or other third party material in this article are included in the article's Creative Commons license, unless indicated otherwise in the credit line; if the material is not included under the Creative Commons license, users will need to obtain permission from the license holder to reproduce the material. To view a copy of this license, visit http://creativecommons.org/licenses/by-nc-nd/4.0/ 